\title{
Heraldo de la Mujer de Ana Roqué: estrategias de posicionamiento en la lucha sufragista puertorriqueña ${ }^{1}$
}

\author{
Ana Roqués Heraldo de la Mujer: Positioning Strategies \\ in Puerto Rican Suffragist Struggle
}

\author{
LUCÍA STECHER GUZMÁN \\ NATALIA CISTERNA JARA \\ Universidad de Chile. Facultad de Filosofía y Humanidades. \\ Correo electrónico: luciastecher@u.uchile.cl \\ Universidad de Chile. Facultad de Filosofía y Humanidades. \\ Correo electrónico: nataliacisterna@u.uchile.cl
}

\begin{abstract}
Este artículo analiza las estrategias de posicionamiento discursivo desplegadas a favor de las demandas ciudadanas de las mujeres y, sobre todo, del derecho al sufragio en el periódico feminista puertorriqueño, Heraldo de la Mujer (1919-1920). La publicación fue fruto del trabajo personal de la intelectual y escritora Ana Roqué y se transformó en un medio a partir del cual la Liga Femínea Puertorriqueńa participó en el debate sobre el derecho al voto de la mujer. Proponemos que en esta publicación se pueden visualizar los mecanismos a través de los cuales Ana Roqué y sus colaboradoras feministas pusieron en circulación e intentaron alcanzar una aceptación amplia de la necesidad de reconocer los derechos ciudadanos de las mujeres. Al mismo tiempo, planteamos que el Heraldo de la Mujer, al ser un proyecto editorial personal de Roqué, constituye un espacio privilegiado para observar las condiciones culturales y materiales que tuvo que enfrentar la intelectual puertorriqueńa para publicar cada uno de los números del medio.
\end{abstract}

Palabras clave: Ana Roqué, Heraldo de la Mujer, sufragismo en Puerto Rico, prensa de mujeres.

This article analyses the discursive strategies deployed by women in Puerto Rican feminist journal Heraldo de la Mujer (1919-1920) in order to further their citizenship rights and specially their right to vote. This publication was mainly the result of the personal work developed by intellectual and

\footnotetext{
${ }^{1}$ Agradecemos al proyecto "Connected Worlds: the Caribbean, Origin of Modern World", en el marco del cual se desarrolla la investigación de la que forma parte este artículo. Este proyecto es financiado por el programa de investigación e innovación de la Unión Europea Horizon 2020 bajo el convenio Maria Sklodowska Curie No 823846. (This project has received funding from the European Union's Horizon 2020 research and innovation programme under the Maria Sklodowska Curie grant agreement No 823846).
} 
writer Ana Roqué. It became the medium through which the "Liga Femínea Puertorriqueña" took part in the debate on women's suffrage. We propose that it is possible to recognize in this journal the mechanisms Ana Roqué and her feminist collaborators used to advance the understanding of the importance of granting women's citizenship rights. At the same time, we argue that being Heraldo de la Mujer mainly a personal editorial project developed by Ana Roqué, it constitutes a privileged space to observe the cultural and material conditions the Puerto Rican intellectual had to face to publish each one of the journal's issues.

Key words: Ana Roqué, Heraldo de la Mujer, Women’s Suffrage in Puerto Rico, Women’s Press.

\section{INTRODUCCIÓN}

Cuenta la crítica Lizabeth Paravisini-Gebert (1991) que Ana Roqué (Aguadilla 1853-Río Piedras 1933), escritora puertorriqueńa que dedicó su vida a la lucha por el sufragio femenino, no aparecía inscrita en ninguna circunscripción para las elecciones de 1932, las primeras en las que las mujeres de Puerto Rico pudieron votar. ${ }^{2}$ Roqué estaba enferma e inválida en ese momento y su mayor deseo era votar por primera vez. En esas circunstancias "sus acompañantes finalmente recurrieron al subterfugio piadoso de hacerle firmar un afidávit, y nunca le dejaron saber que su voto había sido invalidado" (ParavisiniGebert 157). Esta anécdota del final de la vida de Ana Roqué evidencia la centralidad que tuvo para ella la conquista del voto femenino, algo que sabían muy bien quienes la acompañaban en la referida escena electoral.

Ana Roqué murió un año después de esas elecciones, consciente de que gracias a su actividad política -y la de otras feministas sociales como Mercedes Solá, Ana López de Vélez, Isabel Andreu, Beatriz Lasalle, Carmen Gómez, Carlota Matienzo, Luisa Amelia de Hostos, Dolores Pérez Marchand, Elisa Rivera de Díaz, Dolores Piñeiro, Marta Robert, Josefina Villafane, Trinidad Padilla de Sanz y María Cadilla de Martínez, entre otras- las mujeres habían alcanzado el derecho a sufragar (Barceló Miller 1997: 74-75). Roqué se desempeñó en diversas áreas del conocimiento - fue escritora, astrónoma, botánica y fundadora de diversos periódicos- y tuvo siempre entre sus preocupaciones principales el tema de los derechos y la situación de las mujeres. En este artículo nos centraremos en uno de sus proyectos periodísticos, Heraldo de la Mujer, publicado entre 1919 y $1920 .^{3}$ Si bien

\footnotetext{
${ }^{2}$ En 1929 se aprobó en Puerto Rico el derecho al voto de las mujeres que sabían leer y escribir; el derecho universal se convirtió en ley en 1935.

${ }^{3}$ Trabajaremos con los nueve ejemplares digitalizados por el Ayuntamiento de Madrid, disponibles en www. memoriademadrid.es. El primer número disponible es el 20 y el último el 30, pero faltan los números 21 y 26. Aparentemente Heraldo de la Mujer empieza en el número 20 porque, tal como señala la portada de la revista, constituye la continuación del Álbum Puertorriqueño.
} 
en ella participaron en forma más o menos constante otras feministas puertorriqueñas ${ }^{4}$ y en sus primeros números hubo una editora de la versión en inglés -la estadounidense Geraldine Maud Froscher, que vivía en Puerto Rico en la época de publicación del Heraldo de la Mujer-, la lectura de sus números evidencia que se trató sobre todo de un proyecto impulsado y sostenido por Ana Roqué. A través de esta publicación la escritora luchó activamente por la conquista del voto femenino y abrió un espacio para instalar en el espacio público sus perspectivas sobre la situación política de Puerto Rico, noticias del avance de la ciencia y de campańas sufragistas de distintos países.

En un contexto de importantes debates en torno al rol de las mujeres en la sociedad, en el que las demandas de las feministas liberales por la ampliación de la educación y por el acceso al voto eran enfrentadas con discursos conservadores que ponían en duda la capacidad de pensamiento abstracto y racional de las mujeres (La Greca), las publicaciones periódicas fundadas y dirigidas por mujeres como Heraldo de la Mujer, Pluma de mujer, La mujer del siglo XX y Nosotras (Barceló Miller), constituyeron un espacio fundamental para instalar en el espacio público puertorriqueńo los argumentos feministas a favor del derecho a elegir y también a ser electas. En Puerto Rico las sufragistas debieron enfrentarse a la legislatura local, que se negó a hacer valer en la isla la Enmienda diecinueve a la Constitución estadounidense. ${ }^{5}$

La lectura del Heraldo de la Mujer que desarrollamos en este artículo parte de la observación de que la prensa de mujeres, a fines del XIX y principios del XX, constituyó un espacio fundamental para el despliegue de las demandas de los sujetos femeninos. Así, los artículos, crónicas, noticias, ensayos y comentarios reunidos en el Heraldo de la Mujer permiten analizar las estrategias discursivas de las feministas de la época y en particular los esfuerzos de Ana Roqué por instalar su voz en el espacio público. Proponemos que Roqué y sus compañeras eran conscientes de que para tener éxito en el posicionamiento y aceptación de sus demandas debían mostrar que no contradecían totalmente los discursos de género hegemónicos. Sus demandas de acceso al espacio público se sustentaron en la noción de complementariedad de los géneros, de acuerdo con la cual hombres y mujeres aportaban desde sus roles respectivos al progreso de la sociedad. Así, si bien no cuestionaron que la función social de la mujer residía en la maternidad y la conducción del hogar, sí plantearon

\footnotetext{
${ }^{4}$ A partir del número 27, de marzo de 1920, aparece en una de las primeras páginas de la revista una lista de colaboradoras, la que incluye también a cuatro hombres. Las mujeres nombradas escriben desde el primer número del Heraldo de la Mujer, mientras los hombres tienen contribuciones más esporádicas. En la lista de colaboradoras (aparece en femenino a pesar de la inclusión de hombres) reconocemos nombres de importantes feministas y escritoras puertorriqueñas: Trina Padilla de Sanz (la Hija del Caribe), Teresita Mangual de Cestero (Migdalia), Carmela Eulate Sanjurjo, Amparo Fernández Náter, Luisa Amelia de Hostos (Asiul Ailema), A. F. de M. (Ateme), Sisilia Arce de Astol, Carmen García de Quintero (Esmeralda), Conchita Meléndez, María Luisa de Angelis y María Angelina de Hostos. Los hombres son: Dr. Don Manuel Fernández de Juncos, Dr. Don Francisco del Valle Atiles, Dr. Don Cayetano Coll y Toste y Ferdinand R. Cestero.

${ }^{5}$ La Enmienda se propone en 1919 al Congreso de los EE. UU y se ratifica en 1920, con lo que las mujeres estadounidenses acceden al sufragio. La discusión por su formulación y su confirmación coincide con los dos años en los que la publicación de Ana Roqué aparece en Puerto Rico.
} 
que justamente por su capacidad de ejercer esos roles tenían especiales habilidades para ocuparse, en el espacio público, de las leyes que afectaban a niños, ancianos, pobres y enfermos, es decir, de las personas de cuyo cuidado normalmente se hacían cargo. Tomando en cuenta esto nos interesa analizar en el Heraldo de la Mujer la forma en que las feministas reprodujeron y a la vez cuestionaron los discursos de la élite para poder convencer a sus lectores y lectoras del carácter justo de su demanda por derechos ciudadanos.

Los principales argumentos utilizados en el Heraldo de la Mujer para posicionar y habilitar las demandas feministas se articulan en torno a dos ejes ideológicos que tienen una posición hegemónica en la sociedad puertorriqueña de las primeras décadas del siglo XX. Se trata, por un lado, del rol central que juega la maternidad en la vida de las mujeres y de cómo de él depende la constitución de sociedades "moralmente sanas". Por otro lado, la centralidad que adquiere la noción de progreso y la confianza en que las transformaciones impulsadas por las élites permitirían el advenimiento pleno de la modernidad en Puerto Rico. Estos dos ejes aparecen ejemplarmente expresados en uno de los textos de portada de la revista:

Es la mujer, es la madre, la que civiliza a los pueblos.

Dadme madres educadas y virtuosas, y el pueblo será culto y moralizado.

Dadme madres ilustradas, y el pueblo será faro esplendoroso donde brillarán con fulgor inextinguible, las Ciencias, las Artes, la Poesía y la Literatura, ésa (sic) que cultiva los grandes ideales de la Humanidad, y va iluminando los senderos do (sic) la piqueta de la Ciencia va descubriendo los arcanos de lo desconocido (No27, marzo de 1920).

Organizamos este artículo en dos apartados de análisis que buscan mostrar de qué modo se amplían y modifican estos ejes para hacer avanzar las demandas de las mujeres. Para contextualizar el periódico y la figura de Ana Roqué, presentamos en primer lugar información sobre ambos y sobre Puerto Rico durante las primeras décadas del siglo XX.

\section{Ana Roqué y el HeRaldo de la MUJer}

Los estudios dedicados a reconstruir la vida de Ana Roqué se basan, en gran medida, en su breve "Autobiografía". Este texto, escrito en el periodo de aparición del Heraldo de la Mujer, ${ }^{6}$ da cuenta de la importancia que la autora le concedió a las experiencias educativas de las que gozó a lo largo de su vida y que, a su vez, le permitieron contribuir a la educación de mujeres y a la formación de profesores y sobre todo de profesoras. El énfasis de este escrito autobiográfico está puesto en la educación de Roqué, en su desempeño como astrónoma, profesora, botánica y en su capacidad de sustentar económicamente a sus cinco hijos después

\footnotetext{
${ }^{6}$ No está claro en qué circunstancias fue escrita esta autobiografía. Su primera publicación en prensa es de 1953 en la revista Asomante, la que rindió homenaje a Ana Roqué en ocasión de los cien años de su nacimiento.
} 
de separarse de su marido, que había perdido su capital por malos negocios. En un texto en el que predomina la información objetiva en torno a la trayectoria de su autora-sobre todo las fechas clave de su educación formal y de su desempeño como profesora y fundadora de escuelas-, la única anécdota más narrativa que encontramos se refiere a su mudanza a los diecinueve años a la hacienda de su esposo, donde prohibió que se castigara a los esclavos y posteriormente celebró la abolición de la esclavitud a pesar de los perjuicios económicos que le causó al marido. La valoración de la libertad de los esclavos es un elemento importante en la construcción del yo de la "Autobiografía", en la que el rasgo principal, como ya señalamos, es la autonomía de la autora y su voluntad de educarse y educar. Comparado con esto, le dedica muy poco espacio a su experiencia como madre y esposa, temas considerados centrales en la vida de las mujeres.

Paralelamente a la labor docente a la que se dedicó toda su vida, Roqué fundó y dirigió distintos periódicos y lideró el movimiento sufragista puertorriqueño. En 1894 creó La mujer, el primer periódico dirigido por una mujer para un público femenino. Este diario se publicó entre 1894 y 1896 y se enfocó fuertemente en la promoción de una educación integral de la mujer, la que debía prepararla para ejercer una ciudadanía completa. Según María de Fátima Barceló Miller "el discurso feminista brotó en esta revista" (54). En 1896 Roqué dirigió La evolución, trisemanario que apareció durante algunos meses y del que no se conservan ejemplares (Barceló Miller 93-94). Varios años después, y en el contexto de un marcado activismo por el derecho de las mujeres al sufragio, Ana Roqué funda La mujer del siglo XX (1917), que codirige con Mercedes Solá. Debido a que tuvo que mudarse a Humacao, Roqué dejó la dirección de esta revista en manos de Solá, comprometiéndose a fundar un nuevo diario cuando se hubiera instalado en su nueva residencia (Barceló Miller). Un año después Roqué fundó en Humacao el Álbum puertorriqueño, periódico dedicado a temáticas generales. En 1919 la autora rebautiza la publicación como Heraldo de la Mujer y dedica sus páginas a tratar temas feministas. Es posible conjeturar que tras su instalación en Humacao Roqué prefirió empezar sus proyectos periodísticos con un diario dirigido a un público amplio y sin reivindicaciones que pudieran despertar suspicacias.

La mujer del siglo XX y el Heraldo de la Mujer se complementaron en su función de órganos de difusión de los debates que se daban en torno al sufragio femenino, permitiéndole a las mujeres de la Liga Femínea Puertorriqueńa (fundada por Roqué en 1917) defender los proyectos que presentaban a la legislatura de la isla para conseguir el voto femenino. En 1921 la Liga Femínea se convirtió en la Liga Social Sufragista e incluyó entre sus objetivos abogar por el derecho de las mujeres a ocupar escańos políticos.

Es interesante observar que el compromiso con el sufragismo no aparece ni en la "Autobiografía" de Roqué ni en sus cuentos o novelas. Su texto literario más estudiado

\footnotetext{
${ }^{7}$ La alianza entre anti-esclavismo y lucha por los derechos de las mujeres es de larga data y en ella se puede ubicar el surgimiento del movimiento feminista en Estados Unidos (Davis). Cercana como era a los debates de las feministas de Estados Unidos, Ana Roqué tiene que haber estado familiarizada con el origen abolicionista del movimiento feminista y al destacar en su "Autobiografía" su compromiso con la libertad de los esclavos se hace parte de esta tradición.
} 
es Luz y sombra (1903), novela en parte epistolar en la que dos amigas se cuentan sus sueños y problemas matrimoniales. Si bien la novela transgrede diversos elementos de la construcción de la femineidad tradicional (LaGreca 2009, Cisterna y Stecher 2017), sobre todo los referidos a la inexistencia e inaceptabilidad del deseo femenino, su trama gira casi en forma exclusiva en torno al tema del matrimonio y la maternidad. En su literatura Roqué, al igual que otras escritoras decimonónicas y de principios del siglo XX, no tematiza aspectos de su vida profesional y política. Las demandas de las mujeres y las dificultades materiales y económicas que enfrentaban a diario las escritoras no tienen presencia en las ficciones de autoría femenina. ${ }^{8}$

Al contrario de lo que sucede en la narrativa de mujeres de la primera mitad del siglo XX -incluida la de Roqué-, en el Heraldo de la Mujer encontramos numerosas referencias a los desafíos que implica mantener una publicación periódica. En todos los números se solicita a las y los suscriptores a que cumplan con sus responsabilidades de pago (los ejemplares eran enviados a las casas de la élite letrada y se asumía que si no los devolvían se comprometían a pagar por cada número recibido), señalando explícitamente las dificultades que plantea el alto costo de la imprenta. También hay una activa búsqueda de anunciantes y se le concede un espacio destacado - las primeras páginas de la revista- a quienes pagan por él. Por ejemplo, en el número 24, de septiembre de 1919, encontramos avisos que promocionan la próxima publicación de la narrativa de Roqué en dos tomos, así como un breve texto titulado "Atención", en el que se solicita que cancelen la suscripción todas las personas que habiendo recibido la revista no la han devuelto. Se apela incluso a la honra de las personas y la confianza que se había depositado en ellas: "Sólo a personas que gozan de buena reputación por su honradez, y que conocemos, nos decidimos a enviárselo, confiando en que no serán capaces de defraudar una empresa naciente y patriótica, que debe protegerse en todo país civilizado" (4). Más adelante, en el mismo número, se intercalan entre medio de los artículos frases como "Suscríbase a Heraldo de la Mujer, el periódico feminista por excelencia", "Anúnciese en el Heraldo de la Mujer, porque es la mujer la que hace el gasto en el comercio", "Si usted quiere ver prosperar su negocio, anúnciese en este periódico" (10).

Las referencias a las condiciones de producción también evidencian el carácter personal del proyecto editorial del Heraldo de la Mujer. Cuando hay atrasos en la publicación de algún número, las explicaciones y excusas refieren a situaciones personales de Ana Roqué como, por ejemplo, una eventual enfermedad, el cambió de ciudad de la autora, su obligación de cuidar a una hija. ${ }^{9}$ La revista funciona también como espacio de difusión comercial de

\footnotetext{
${ }^{8}$ Sobre la escasa representación de las experiencias de las autoras en su mundo cultural y profesional, y la nula referencia a las condiciones materiales en las que estas actividades se desarrollaban, ver Natalia Cisterna (2016).

${ }^{9}$ En una de las entradas de la revista titulada "A nuestros suscriptores y anunciantes" se señala que el periódico solo se empezará a publicar en espańol y que saldrá una vez al mes. La explicación de estos cambios en el formato y la periodicidad es que se vieron "afectadas" por una enfermedad. La persona en plural retóricamente elude al sujeto individual, destaca el carácter colectivo de la publicación, pero en este como en otros apartados resulta evidente que la persona que se enfermó -o enfrentó otras dificultades que afectaron la publicación- es Ana Roqué.
} 
las publicaciones literarias de la escritora y de su trabajo como maestra (aparecen anuncios con los precios de las novelas de la autora y también con ofertas de clases de aritmética y álgebra para puertorriqueńos y de castellano para estadounidenses). La revista muestra, sin ambages, la energía que le dedicó Roqué a este proyecto periodístico y las dificultades que atravesó para mantenerlo y para ganarse la vida. Estas explican, en gran medida, la vida relativamente corta de muchas de las publicaciones periódicas de la época, sobre todo las impulsadas por mujeres que contaban con menos recursos y apoyo.

\section{LA MATERNIDAD, EL MATRIMONIO Y LAS DEMANDAS CIUDADANAS}

Como señalamos al inicio de este artículo, uno de los discursos hegemónicos que las mujeres de la primera mitad del siglo XX no cuestionaron es el referido a la centralidad de su función social como madres y esposas. La maternidad sigue apareciendo en sus escritos como el rol que indiscutiblemente deben cumplir y que da sentido a sus vidas. Con respecto a esto, es importante recordar que a lo largo del siglo XIX y también a principios del XX las mujeres en América Latina apelaron a su función maternal para demandar educación. Y los hombres estuvieron de acuerdo en que la formación de los futuros ciudadanos de las naciones latinoamericanas requería de madres libres de creencias supersticiosas ${ }^{10}$ y capaces de transmitir a sus hijos algunos contenidos educativos mínimos. En el Heraldo de la Mujer encontramos que ese argumento también fue usado en la lucha por el derecho a votar y a ocupar cargos de elección popular en la legislatura. Como mencionamos anteriormente, se aducía que el rol maternal y las responsabilidades de cuidado que asumían las mujeres las habilitaban especialmente para legislar en materias concernientes a los grupos sociales más vulnerables.

$\mathrm{Al}$ conceder que el principal aporte a sus naciones lo podían hacer desde su rol de madres y esposas, las feministas puertorriqueñas y latinoamericanas no problematizaron la identificación de las mujeres con la maternidad y la crianza. Sin embargo, muchas veces transgredieron los límites entre lo público y lo privado, utilizando argumentos sustentados en un orden de género que, paradójicamente, al mismo tiempo era subvertido. Si en virtud del saber adquirido en el manejo de asuntos domésticos de vital importancia para la sociedad -como la educación de los niños y el cuidado de los grupos vulnerables- las mujeres resultaban ser las que mejor podían legislar sobre ellos, su confinamiento en el mundo privado dejaba de tener sentido para la sociedad.

En la prensa de mujeres encontramos no solo un conjunto de esfuerzos por ampliar los espacios de participación femenina, sino también la configuración de una esfera de encuentro colectivo, forjado a partir de las redes de solidaridad y cooperación intelectual

\footnotetext{
${ }^{10}$ Esto aparece con claridad en un texto tan temprano para la historia literaria y política latinoamericana como El Periquillo Sarniento (1816) de Fernández de Lizardi. En esta novela, el protagonista censura las creencias supersticiosas en las que su madre basó su educación. Su padre, por el contrario, representaba un saber ilustrado razonable, mucho más apropiado para orientar la vida de un muchacho.
} 
que trascienden las fronteras nacionales. Tal como seńala Mary Louise Pratt (1993), para las mujeres latinoamericanas los vínculos con sus pares de otros países fueron fundamentales en la articulación de discursos a favor de sus derechos:

Como habitantes sin ser plenamente miembros de las naciones, las mujeres que han tenido acceso a la esfera pública se han comprometido críticamente con los hábitos de pensamiento de los imaginarios nacionales. En la política, los movimientos femeninos autónomos han sido mas frecuentemente internacionalistas y antimilitaristas, por ejemplo. En las letras, al menos antes de 1945, las intelectuales latinoamericanas pensaban no en términos de patrimonios nacionales (que típicamente han excluido su trabajo), sino más bien en términos mundiales o hemisféricos. Actuando como escritoras, comentadoras, editoras de revistas, traductoras, antologistas y mentoras, muchas enfocaron su trabajo cultural en prácticas de lo que se podría llamar mediación trasnacional (Pratt 55).

Este carácter transnacional de los vínculos entre mujeres permea todos los números del Heraldo de la Mujer. En ellos encontramos permanentemente noticias sobre los avances de las sufragistas en el mundo, artículos de escritoras de otros países reproducidos en su interior, agradecimientos a otros medios por haber publicado textos de autoras del Heraldo de la Mujer, referencias a reuniones, encuentros, congresos de mujeres que trabajan en conjunto para mejorar su situación.

Uno de los efectos de esos vínculos transnacionales-textuales y también presenciales a través de viajes y encuentros en distintos países- es que permiten confrontar el aislamiento que caracteriza a una de las figuras típicamente asociada a la domesticidad femenina: la del ángel del hogar. Este concepto proviene del poema narrativo "The Angel of the House" del inglés Coventry Patmore, publicado por primera vez en 1854, pero los atributos de la mujer que en él se proclaman aparecen ya en libros como La perfecta casada de Fray Luis de León (1584). En España, América Latina y el Caribe tiene gran difusión el tratado de conducta $E l$ ángel del hogar (1859) de la escritora conservadora Maria del Pilar Sinués (LaGreca).

En "Profesiones para las mujeres", Virginia Woolf afirma que para poder escribir lo primero que debe hacer la mujer es matar al ángel del hogar. Este configura un modelo de mujer estructurado en torno a la abnegación, la dedicación al marido y los hijos, por lo que resultaba totalmente paralizante para cualquier esfuerzo por expresarse a través de la creación artística. Una mujer sin deseo propio, subordinada totalmente a la voluntad del marido y pendiente de las necesidades del resto difícilmente puede tener un proyecto creativo (La Greca, Gilbert y Gubar 1998).

Si bien el Heraldo de la Mujer participa del discurso de celebración del rol maternal de las mujeres, también enfrenta directamente el modelo del ángel del hogar. Dos textos son especialmente interesantes en este sentido. En el número 22, de abril de 1919, la sección "Plumazos" firmada por Lotys -que tenemos buenos motivos para creer que es un seudónimo de Ana Roqué- comenta una nueva derrota, propinada por los miembros de la 
Cámara, a la lucha de las mujeres por obtener el voto. En esta nota se menciona la imagen del ángel del hogar, caricaturizándola y burlándose de la visión de los hombres que integran la clase política, para quienes el feminismo sería un conjunto de gestos galantes, asociados a la idea tradicional de "respeto" a las mujeres, que en ningún caso contempla la concesión de derechos políticos: "Por fin, por fin derrotaron, en nuestra flamante Cámara el voto de la mujer. Las palomas para el nido; el león para el combate; y el Progreso, cual inútil chirimbolo, contra un estante (...). Pero eso sí, son nuestros feministas muy coreteros. Mucho de ángel bello, de hada primorosa y de sol divino; $y$ de flor preciosa de los jardines del cielo..." (21, destacado en el original).

En un artículo más extenso, aparecido en el número 23 de 1919, Ana Roqué escribe un homenaje a dos ilustres escritores puertorriqueños, Luis Muñoz Rivera y José de Diego. Con respecto a ambos la autora destaca la calidad de sus versos y su amor por Puerto Rico. En relación con José de Diego, a la vez que lo alaba le critica el haber dedicado su poesía a un tipo de mujer que existe solo en la fantasía masculina:

José de Diego habiendo pasado gran parte de su vida en aquella Europa, fuertemente saturada, hasta ha poco, de prejuicios medioevales (sic); y exaltada su imaginación por ese lirismo del cruzado, que rompía lanzas por su patria y por su dama; era feminista a su manera, rindiendo culto idolátrico a la mujer ideal, a la mujer santa, a la mujer ángel, a la mujer que, desgraciadamente, solo existe en su imaginación de poeta: a la mujer diosa de los sueños y leyendas, utopía de un pasado que se esfumó con el romanticismo; sin volver los ojos jamás a la mujer moderna, a la mujer de nuestro días, que ora trabajando, que piensa, que calcula, que vive su vida de lucha y de dolor (5, destacado en el original).

En este artículo, y en particular a través de esta cita, podemos observar la estrategia de Ana Roqué de desarrollar una postura crítica a la concepción de la mujer que construyen los poetas -en este caso José de Diego- en el marco de un artículo destinado a elogiar su obra (el título refuerza ese sentido: "Dos cumbres. Una flor del recuerdo para nuestros muertos ilustres. Muñoz Rivera, José de Diego"). A la inexistente mujer ángel, Ana Roqué contrapone la mujer moderna, sobre la cual subraya que "ora trabajando", es decir, cumple con sus deberes religiosos a la vez que se desempeña en el mundo laboral.

\section{Sobre El PROgReso MOdERNO Y EL SUFRAGio FEMENINO}

Los números que se conservan del Heraldo de la Mujer llevan el siguiente lema: "Las autocracias y democracias-a medias-, están condenadas a desaparecer, barridas por el humano progreso". El mensaje que acompaña al título de la publicación comulga ideológicamente con la convicción de la elite letrada del periodo que cree en una modernidad que avanza inexorablemente, y en que los regímenes que la niegan o aplican sus principios de forma 
timorata están destinados a desaparecer. En la línea editorial del Heraldo de la Mujer, una "democracia a medias" es la que deja fuera de su desarrollo político y social a las mujeres, esas "democracias" imperfectas, por tanto, tienen su destino sellado. Lo interesante de este lema es que no es simplemente una consigna con un mensaje efectista, sino que revela una estrategia política que vemos reproducida en distintos apartados de la publicación de Ana Roqué, y que consiste en instalar la igualdad de la mujer como una demanda que trasciende a sus directas involucradas, para impactar en el corazón mismo del proyecto moderno, favoreciendo su materialización.

Desde el siglo XIX, el progreso moderno se constituyó para las elites latinoamericanas y caribeńas en el discurso hegemónico (Goldgel 2013), arraigando en ellas la certeza de que el futuro deparaba mayores niveles de desarrollo social y tecnológico. Se podía poner en duda el tipo de esfuerzos que desplegaban las clases dirigentes para alcanzar este anhelo modernizador, pero no se problematizaba ni discutía su carácter irrevocable. Los debates se centraron, entonces, en identificar las prácticas, costumbres, discursos y grupos sociales que dificultaban el "avance natural de la historia" y que retrasaban el futuro. Así, la "barbarie" asociada a comunidades originarias, sectores populares, negros, mestizos, zambos y mujeres, fue identificada como el principal obstáculo para lograr la superación de la empantanada situación en que habían quedado nuestros pueblos después del largo periodo colonial. En estos segmentos sociales arraigaban las fuerzas retardatarias que debían ser superadas, y para ello los grupos dirigentes configuraron discursos que representaban, describían y explicaban cómo estos sectores impedían el desarrollo moderno, al tiempo que diseńaban políticas y propuestas destinadas a restringir su participación en la esfera pública. Para el caso de las mujeres, su contribución social se definió en los parámetros de la ideología doméstica. En la medida, se decía, que no estaban naturalmente capacitadas para hacerse cargo de tareas productivas en la esfera social, su verdadero aporte consistía en colaborar en la constitución de un espacio hogareńo funcional al proyecto moderno: trasmitiendo los valores ciudadanos en el seno familiar y haciéndose cargo de labores asistenciales. Para ellas, la modernidad se redujo, entonces, a ejercer de manera profesional y eficiente su rol doméstico dentro del hogar.

Al respecto, la línea editorial del Heraldo de la Mujer recoge los postulados fundamentales de las feministas puertorriqueñas del periodo, cuestionando esta definición restringida y distorsionada de entender la modernidad del sujeto femenino. Para Ana Roqué y las colaboradoras de la publicación, el progreso moderno no se ve amenazado ni entorpecido por el ingreso de mujeres autónomas al mundo público, sino por la persistencia de un discurso que las confina a tareas domésticas y no advierte los costos de seguir prescindiendo de su fuerza laboral e intelectual. En el Heraldo de la Mujer estos principios se expresan en dos planos: por una parte, conectando la noción de progreso con la de sujeto femenino moderno. Desde su estructura y la selección de temas y notas, el periódico despliega la idea de que el futuro está asociado a una nueva forma de constitución del género femenino. Por otra parte, el periódico enarbola una demanda inmediata del movimiento de mujeres: el voto femenino. Las columnas de la revista exponen y discuten las distintas etapas del debate 
político en Puerto Rico en torno al sufragio y plantean el carácter necesario del mismo para la profundización de la democracia.

La identificación entre progreso y sujeto femenino moderno se manifiesta desde el formato general del periódico con una organización de sus contenidos que trasmite la idea de un avance permanente: las y los lectoras/es se enfrentan a un periódico feminista que las/ os sumerge en un mundo marcado por un ritmo acelerado de sucesos y cambios constantes, entregando la sensación de que el futuro es un lugar cada vez más próximo. En este sentido, creemos que la función ideologizadora del periódico se sostiene en buena medida no solo en los contenidos explícitos a favor de los derechos de la mujer, sino también en la forma de comunicarlos, para lo cual es imprescindible conocer los gustos y hábitos de lectura del público al que el medio apunta. Al revisar el Heraldo de la Mujer, se hace evidente que su editora busca llegar a una base amplia de lectores/as, lo que queda de manifiesto en el artículo del número 24 del periódico, "Gramática Castellana Janer", firmado por la propia Ana Roqué, en donde junto con elogiar la edición del libro que da título a la columna, reflexiona sobre el lenguaje de la prensa, dando cuenta de que en el Heraldo de la Mujer la forma escritural no es algo menor y requiere estar despojada de términos alejados del uso común: "Nosotros que nos dedicamos a escribir para el público, no tenemos inconvenientes en usar las palabras debut, susceptible, accidentado, reporter, record y otras que matizan hoy nuestro lenguaje ... Si fuéramos a usar el clásico lenguaje castellano en nuestros escritos, resultarían pesados y con cierto sabor arcaico" (9).

La necesidad de llegar a públicos amplios se evidencia, además, en los diversos asuntos que aborda la revista, como la autonomía política de Puerto Rico, novedades científicas, literarias y noticias del contexto internacional, teniendo especial tribuna, por supuesto, los debates sobre el voto de la mujer y sus derechos ciudadanos. Esta heterogeneidad de temas se presenta agrupada en distintas notas cortas en las secciones "Mundial", "Social" y "Sección científica", o de manera autónoma en formatos más extensos, pero que rara vez sobrepasan una página. La acotada extensión de los escritos, su lenguaje directo y en ocasiones coloquial e irónico, como el usado en la sección "Plumazos", facilita la rápida comprensión de las columnas y notas del Heraldo de la Mujer en públicos modernos cada vez más sujetos al reloj y ocupados por proyectos y responsabilidades diversas.

En el Heraldo de la Mujer la variedad de temas tratados configura un mapa acrisolado de acontecimientos que favorecerá el vínculo que se desea proyectar entre el movimiento de mujeres y el desarrollo moderno. Así, la nota sobre el vuelo de un aeroplano que recorre Mineola a gran velocidad junto a un texto que refiere al estado actual del activismo feminista español (núm. 20), o bien la noticia de la aparición de nuevos periódicos de sufragistas italianas al lado de aventuradas teorías científicas sobre el impacto que tiene la alineación de los planetas en los sismos (núm. 25), forman la imagen de una escena local y mundial conectada, en la que las conquistas de las mujeres y sus luchas por la igualdad, tanto en Puerto Rico como en el resto de los países, más los adelantos técnicos y los sucesos de interés mundial, son parte de un mismo relato que no deja de escribirse. En otras palabras, formalmente el diario opera como una suerte de aleph que permite observar al mismo 
tiempo un panorama nacional e internacional en que el progreso se asocia a la emergencia del sufragio femenino y a la participación de la mujer en el ámbito público.

En el Heraldo de la Mujer el sufragio femenino es la demanda política inmediata que concentra gran parte de las columnas y notas sobre los derechos ciudadanos de la mujer. El carácter urgente que adquiere el tema en la publicación no solo se explica por la sintonía del periódico con las luchas que están realizando los distintos movimientos sufragistas a nivel mundial, sino también por el contexto específico de Puerto Rico, en donde la discusión del derecho al voto de la mujer involucra la compleja relación de dependencia de la isla con los EE. UU. Como señalamos al inicio del artículo, el Heraldo de la Mujer surge en medio de un encendido debate sobre el voto femenino en una coyuntura especial: la aprobación de la Enmienda diecinueve (1920) a la Constitución de los EE. UU., en la que se indica que no se puede denegar el derecho al voto a ningún ciudadano por su sexo. Desde 1917, a partir de la Enmienda Jones, los puertorriqueños tienen la ciudadanía estadounidense, lo que permite que la modificación constitucional que concede derechos a las mujeres pueda aplicarse también en la isla. Al menos así lo pensaron las feministas que vieron en la firma de la Enmienda una oportunidad única para conquistar un derecho básico del quehacer ciudadano. Sin embargo, los poderes políticos locales no validan la Enmienda en Puerto Rico, lo que genera el estupor de las feministas de la isla y un intenso debate político. El Heraldo de la Mujer entrará en este debate de manera directa. Para ello desplegará dos modalidades discursivas: notas breves en las que se muestra el avance del voto femenino en el resto del mundo y el estado de la discusión en Puerto Rico, y columnas de opinión en las que se reflexiona sobre el voto femenino y las posibilidades que abre la Enmienda estadounidense.

Las notas se despliegan, principalmente, en las secciones "Mundial" y "Social" y recogen diversas noticias sobre el movimiento sufragista en distintas latitudes: desde el exitoso desarrollo de congresos de mujeres por sus derechos civiles en Europa, hasta la obtención del voto femenino en países como Filipinas o Italia. El estilo magazinesco de la publicación es especialmente claro en estos apartados, cuyo carácter híbrido se evidencia en la coexistencia de crónicas de matrimonios de las clases acomodadas, con saludos a viajeros que las redactoras conocen, denuncias de sucesos que afectan a la población y posicionamientos con respecto a la educación de las mujeres y el feminismo.

A pesar de que la mayor parte de las notas son cápsulas de información, en algunas de ellas encontramos un análisis más elaborado que sobrepasa la mera función de mantener al día a su público. Es lo que se puede leer, por ejemplo, en el texto "El feminismo y la religión", del núm. 22, que discute una tesis aparecida en el medio liberal El amigo de todos, en la que señala que "El feminismo no debe existir sin religión" (20). El Heraldo de la Mujer responde con la afirmación de que el feminismo es una aspiración política de igualdad para las mujeres y nada tiene que ver con la religión. El texto pone de manifiesto la conciencia de las feministas del Heraldo de la Mujer del rol político que tiene el movimiento de mujeres y la importancia de separar la demanda por sus derechos civiles, de otro tipo de discursos y de espacios que no corresponden al debate. En el número siguiente este grado de conocimiento 
que exhiben las feministas sobre la praxis política, y sus específicos radios de acción, se hace extensivo a las mujeres en general. El texto "Opinión valiosa de un republicano" (15), cita la opinión de un ciudadano (al que no se identifica) que escribe al periódico y expresa estar a favor del voto femenino. Se destaca en el escrito que la mujer puertorriqueña está más preparada para ejercer cargos políticos que los varones y que, por lo mismo, debería tener plenos derechos ciudadanos. Se usa, así, la estrategia de poner en las elogiosas palabras de un varón, el reconocimiento de las capacidades políticas del sujeto femenino. La voz del emisor aparece validada por su condición de "republicano", es decir, de ciudadano interesado en el fortalecimiento de la democracia y el imperio de la justicia.

En la nota "Las mujeres italianas pueden votar", del núm. 25, se señala que el voto femenino no solo sería favorable para la sociedad porque la mujer puede hacerse cargo de importantes tareas políticas, sino porque el reconocimiento de sus derechos estimula en ella una mayor entrega y espíritu de sacrificio por la nación:

Leemos en nuestra interesante colega Voce Nuova, de Milán, que las mujeres italianas satisfechas porque esa nación progresista les haya concedido el derecho del sufragio, se proponen propender a su engrandecimiento y han tomado a su cargo a trabajar por extirpar el analfabetismo, y proteger la nińez desvalida. Lo mismo se proponen hacer las señoras españolas, las cuales han aceptado el reconocimiento de sus derechos con la mayor alegría, desde la más encumbrada duquesa, y la más encopetada de la clase media, hasta las progresistas obreras de las fábricas. El mundo marcha.... ¿Qué dicen a esto las puertorriqueñas? (11).

En la cita nuevamente queda clara la imagen de un mundo que avanza, pero esta vez no solo más rápido, sino que de mejor manera gracias a los derechos concedidos a las mujeres. La pregunta final dirigida a las puertorriqueñas tiene como función depositar en las compatriotas, a veces poco involucradas en la lucha por sus derechos, la responsabilidad de hacerse parte de una escena de movilización feminista que solo puede traer beneficios a la totalidad de la nación.

Las polémicas no quedan fuera de esta tribuna periodística. A partir de ellas el periódico puede trasmitir la intensidad y la urgencia que tiene en el ámbito público la discusión sobre el voto para la mujer y exponer los débiles argumentos de sus detractores. La nota "Contestación al Sr. Miguel Guerra", del núm. 22, es un claro ejemplo de este tipo de discusiones. Firmada por La Redacción ${ }^{11}$, la respuesta interpela al congresista puertorriqueño que en la Cámara había seńalado que la Legislatura de la isla no puede autorizar el voto femenino y, por tanto, no era aplicable la Enmienda diecinueve. En su contestación, La Redacción del Heraldo de la Mujer advierte a Guerra que se enfrenta a mujeres informadas

\footnotetext{
${ }^{11}$ Suponemos que la mayoría de los escritos firmados por La Redacción fueron elaborados por Ana Roqué. Al ser ella la editora y principal columnista del periódico, no es extraño que recurriera a esta nominación más impersonal para firmar sus aportes al diario.
} 
en el campo de las leyes y la política y con capacidad de movilizar a distintos sectores a favor de su causa: "[Miguel Guerra] imaginó que la mujer puertorriqueńa doblaría humildemente la cabeza y se sometía a esa decisión como un hecho cierto. Pero la mujer puertorriqueña está completamente decidida a defender su derecho, ahora y siempre" (55, destacado en el original).

Como revisábamos en el apartado anterior, no es la primera vez que en el Heraldo de la Mujer se muestra la distancia que existe entre el modelo de mujer (ángel del hogar), presente solo en los argumentos de los varones de los círculos letrados, y la "mujer real", definida por la publicación como una sujeto dispuesta a encarar desafíos en el espacio público y a pelear por sus derechos. En la respuesta a Miguel Guerra, La Redacción se asume como portavoz de esa mujer puertorriqueńa políticamente informada y subestimada por el político local. El mensaje es evidente: mientras el Heraldo de la Mujer representa los intereses de la mujer puertorriqueña, Guerra habla sobre una mujer que solo existe en su imaginación. Al mismo tiempo, la nota muestra una dimensión política interesante: hace gala de la capacidad del medio de Ana Roqué para establecer contactos con circuitos importantes de la escena política estadounidense. En efecto, la contestación a Guerra viene apoyada con una carta del senador estadounidense Miles Poindexter, quien confirma que las mujeres en Puerto Rico se verían beneficiadas por la Enmienda que se discute en los EE. UU. La carta, se indica, ha sido conseguida por Helen H. Gardener del Comité Congresional de la Asociación Nacional Sufragista de la Mujer Americana, y es ella la que la envía al periódico puertorriqueño. Es destacable que el Heraldo de la Mujer en una nota corta que tiene como fin refutar los argumentos puntuales de Miguel Guerra, dedique espacio a exponer la cadena de comunicación que permite obtener la epístola del Senador, evidenciando con ello las conexiones que tienen las feministas de la publicación con las sufragistas estadounidenses, y las de estas con el poder político de su país. Sin duda la "Contestación al Sr. Miguel Guerra" es un texto revelador que posibilita observar cómo el Heraldo de la Mujer escenifica la efectividad de sus redes y la habilidad para poner en movimiento una serie de contactos con el fin de apoyar sus argumentos. La nota, en resumen, pone de manifiesto el ejercicio político profesional de las feministas que editan la publicación y las instala como interlocutoras a la altura de una discusión legislativa.

En cuanto a las columnas de mayor extensión que abordan el tema del sufragio femenino y la participación política de la mujer, cabe destacar el texto firmado por La Redacción, “'Será ‘Buena fe'?”, en el núm. 23. La columna, a partir de una serie de preguntas retóricas, presentes desde el título, critica el rechazo a la Enmienda diecinueve, lo que le niega el derecho al voto a las puertorriqueñas. La argumentación parte citando un párrafo aparecido en el periódico puertorriqueño El Mundo, en el que se señala: "Los pueblos sufren y retrasan el desenvolvimiento de su educación cívica, cuando al tratarse de sus asuntos políticos, aparece "la mala fe"” (6). Tomando esta idea, La Redacción plantea que no hay mejor ejemplo del uso de la "mala fe" en política que lo que está ocurriendo con la negación de la Enmienda estadounidense en Puerto Rico. Cómo explicar, entonces, se pregunta el periódico de Ana Roqué, que se pasen por alto los derechos concedidos en los EE. UU a las 
mujeres y se viole con ello el cumplimiento de la Enmienda Jones que concede la ciudadanía estadounidense a los puertorriqueños. Obviamente, para La Redacción, no es precisamente un acto de "buena fe" lo que lleva a los congresistas locales a tomar esa medida, sino lo que El Mundo identifica como "mala fe", una mala práctica del ejercicio del poder con las inevitables consecuencias que esto trae. Uno de los aspectos más destacables del artículo es que vaticina el desastroso futuro político que le espera a Puerto Rico por negarse a conceder derechos ciudadanos a las mujeres: “ ... así el pueblo puertorriqueño, por haber arrebatado su derecho a la mitad de su propio pueblo, vivirá sometido eternamente a otro pueblo, sin soberanía ni ciudadanía propia, hasta que reconozca su pecado de lesa humanidad, y repare, en lo posible (si pudiera tal acto de despojo injusto, ser reparado algún día) la terrible injustica cometida contra nosotras" (6, destacado en el original).

En un interesante planteamiento retórico, en la columna la no validación de la Enmienda estadounidense por parte de Puerto Rico, lejos de ser un gesto de autonomía frente a las decisiones políticas tomadas por el Congreso de los EE. UU, terminará hundiendo más a la isla en una relación de dependencia permanente. Porque, volviendo al lema que encabeza el título del Heraldo de la Mujer, los estados autoritarios y no democráticos son los que realmente están destinados a desaparecer, viendo diluida su independencia en una relación de subordinación colonial que limita su crecimiento.

\section{Conclusiones}

Los textos literarios de mujeres latinoamericanas y caribeñas de la primera mitad del siglo XX, en general brindan escasa información sobre las condiciones materiales y el circuito de relaciones que facilitaban o dificultaban su ejercicio escritural. La obra de Ana Roqué no es una excepción. Su literatura no ofrece imágenes que simbolicen su instalación y la de sus pares en su campo cultural y político. En ella no encontramos referencias al arduo trabajo de su autora como intelectual y como una de las feministas caribeńas más importantes del periodo.

A diferencia de las obras literarias, la prensa necesita desplegar estrategias directas que le permitan captar lectores que hagan posible su continuidad. Desde anuncios publicitarios, hasta avisos para obtener suscriptores o editoriales que informan de las colaboraciones, la prensa, en especial la de mujeres, constituye un objeto de estudio privilegiado para comprender cómo se desarrolló la labor política y cultural de los sujetos femeninos que se aventuraban a desafiar la lógica de las esferas separadas de acuerdo con el orden de sexogénero.

El Heraldo de la Mujer se publica en un momento crucial de la lucha por el voto femenino en Puerto Rico y el mundo y se constituye en un espacio que permite revisar las distintas aristas que toma el debate sobre el sufragio, que en ese país no estuvo ajeno a la particular situación política de dependencia con los EE. UU. Como señalamos, el Heraldo de la Mujer es, además, un proyecto personal, que en buena medida depende de Ana Roqué. 
En tal sentido, nos permite conocer los esfuerzos que hizo para mantenerlo a flote y las dificultades económicas y logísticas que experimentó en su proceso de edición.

Un aspecto de importancia en el Heraldo de la Mujer son las estrategias desplegadas para instalar el discurso de la igualdad de la mujer y la necesidad de otorgarle derechos políticos y, especialmente, lograr su aprobación de manera amplia. El periódico de Ana Roqué sustenta sus principales argumentos apoyándose en algunos postulados hegemónicos que no se discuten y que, por el contrario, son instrumentalizados para favorecer la aceptación de sus demandas. Los dos ejes hegemónicos en torno a los cuales se articula el Heraldo de la Mujer son, por un lado, el carácter central que adquiere la familia y la maternidad en la vida de la mujer y en la consolidación de la república moderna, y, por otra, la confianza en la modernización y el progreso como destino inevitable para las repúblicas. Desde la línea editorial del Heraldo de la Mujer, como también en el variado corpus de notas y columnas que integran sus números, estos ejes se instrumentalizan en dos argumentos centrales: en primer lugar, la maternidad le brinda a la mujer un acervo de conocimientos sobre la niñez y la crianza que le permitiría participar, con más propiedad que los varones, en el debate legislativo sobre estos temas y, así, contribuir al desarrollo nacional. En segundo lugar, la publicación de Ana Roqué sostiene la idea de que el progreso y la modernidad dependen de la concesión de derechos ciudadanos a la mujer. En cada uno de los números del Heraldo de la Mujer la igualdad de la mujer es parte de un proyecto transformador que culminará en una sociedad propiamente moderna. La lucha por el sufragio femenino, en este sentido, se constituye en una lucha por el futuro.

\section{OBRAS CITADAS}

Armstrong, Nancy. 1991. Deseo y ficción doméstica. Una historia politica de la novela. Madrid: Cátedra.

Barceló Miller, María de Fátima. 1997. La lucha por el sufragio femenino en Puerto Rico. 1896-1935. San Juan de Puerto Rico: Ediciones Huracán.

Cisterna, Natalia y Lucía Stecher. 2017. “Diálogos textuales y personales: Carmela Eulate Sanjurjo, Ana Roqué de Duprey y el feminismo puertorriqueño a principios del siglo XX”. Anclajes 3: 25-41.

Cisterna, Natalia. 2016. "Ceremonias letradas: representaciones del campo cultural en la narrativa de autoras latinoamericanas y caribeńas de la primera mitad del siglo XX". Taller de Letras 59: 151-167.

Davis, Angela. 1981. Women, Race and Class. New York: Random House.

Fernández de Lizardi, José Joaquín. 2018. El Periquillo Sarniento. Cervantes virtual. Septiembre. Web. 5 de sept. de 2018.

Gilbert, Sandra y Susan Gubar. 1998. La loca del desván. La escritora y la imaginación literaria del siglo XIX. Madrid: Cátedra.

Goldgel, Víctor. 2013. Cuando lo nuevo conquistó América. Prensa, moda y literatura en el 
siglo XIX. Buenos Aires: Siglo XXI.

Heraldo de la Mujer (1919-1920). Biblioteca Digital. Memoria de Madrid.

LaGreca, Nancy. 2009. Rewriting Womanhood. Feminism, Subjectivity, and the Angel of the House in the Latin American Novel, 1887-1903. Pennsylvania: The Pennsylvania State University Press.

Paravisini-Gebert, Lizabeth. 1991. "Esquema biográfico." Ana Roqué. Luz y sombra. Río Piedras: Universidad de Puerto Rico. 151-174.

Pratt, Mary Louise. 1993. "Las mujeres y el imaginario nacional en el siglo XIX". Revista de Crítica Literaria Latinoamericana 38: 51-62.

Roqué, Ana. 2018. "Autobiografía de Ana Roqué de Duprey", versión digitalizada de manuscrito mecanografiado. Biblioteca digital puertorriqueña. Web. 5 de sept. de 2018. Roqué, Ana. 1991. Luz y sombra. Río Piedras: Universidad de Puerto Rico.

Showalter, Elaine. 2001. "La crítica feminista en el desierto". Otramente: lectura y escritura feminista. Ed. Mariana Fe. México: F. C. E. 2001. 75-111.

Woolf, Virginia. 2018. "Professions for Women". Tripod. Web. 
